\title{
Caracterización productiva del aguacate (Persea americana) en la zona de alta montaña Veracruz, México
}

\section{Productive characterization of avocado (Persea americana Mill.) in the high mountain area Veracruz, Mexico}

\author{
Nataren-Velazquez, Jeremías; Angel-Pérez, Ana Lid del; Megchún- \\ García, Juan Valente; Ramirez-Herrera, Eréndira; Meneses-Marquez, \\ Isaac; Editor Academico Prof. Dr. Carlos Alberto Zúniga-González
}

\author{
iD Jeremías Nataren-Velazquez \\ nataren.jeremias@inifap.gob.mx \\ Instituto Nacional de Investigaciones Forestales, \\ Agrícolas y Pecuarias, México \\ iD Ana Lid del Angel-Pérez \\ angel.analid@inifap.gob.mx \\ Instituto Nacional de Investigaciones Forestales, \\ Agrícolas y Pecuarias, México \\ (iD Juan Valente Megchún-García \\ juanmg_3@hotmail.com \\ Instituto Nacional de Investigaciones Forestales, \\ Agrícolas y Pecuarias, México \\ (iD) Eréndira Ramirez-Herrera \\ erendiher@gmail.com \\ Instituto Nacional de Investigaciones Forestales, \\ Agrícolas y Pecuarias., México \\ (iD Isaac Meneses-Marquez \\ meneses.isaac@inifap.gob.mx \\ Instituto Nacional de Investigaciones Forestales, \\ Agrícolas y Pecuarias. , México \\ Editor Academico Prof. Dr. Carlos Alberto Zúniga- \\ González \\ Universidad Nacional Autónoma de Nicaragua, \\ León.Escuela de ciencias agrarias y veterinarias. \\ Departamento de Agroecología., Nicaragua
}

Revista Iberoamericana de Bioeconomía y Cambio Climático

Universidad Nacional Autónoma de Nicaragua, León, Nicaragua ISSN-e: 2410-7980

Periodicidad: Semestral

vol. 6, núm. 12,2020

czuniga@ev.unanleon.edu.ni

Recepción: 03 Julio 2020

Aprobación: 02 Octubre 2020

URL: http://portal.amelica.org/ameli/ jatsRepo/394/3941759001/index.htm
Resumen: México es uno los países con mayor producción de aguacate en el mundo y la variedad mayormente plantada es la Hass, además que Michoacán, México figura como el principal productor de aguacate con un $80 \%$ seguido de otros estados como Jalisco, estado de México. En Veracruz existe una superficie plantada en la parte central del estado que necesita ser caracterizado. El objetivo de esta investigación es tipificar la zona productora de aguacate en la zona de alta montaña de Veracruz, México. La zona de productora de aguacate en la región de altas montañas, Veracruz, México, está integrado por los municipios de Alpatlahua, Calcahualco, Coscomatepex e Ixhuatlan del café, lugar donde se aplicó una encuesta entrevistando a productores a través de una entrevista con el instrumento denominada como cuestionario semiestructurado con preguntas abiertas y cerradas; se realizó el análisis de la información de manera sistemática con análisis descriptivo y multivariado de componentes y correspondencia, además, para algunas variables se realizó análisis paramétrico y no paramétricos de los datos. Los resultados obtenidos agrupan a productores pequeños, con el $80 \%$ de hombres y el resto integrados por mujeres; la mayoría de los productores saben leer y escribir, también se observó que se dedican a otras actividades diferentes al cultivo de aguacate. El productor aguacatero posee conocimiento tácito en el manejo del cultivo desde la etapa plantación hasta la producción del cultivo. Los productores poseen experiencia en el manejo de otros cultivos, esto es porque tiene la finalidad de obtener otros ingresos adicionales.

Palabras clave: Persea americana, Frutales, Diagnóstico de cultivo.
Abstract: Mexico is one of the leading countries in avocado production worldwide and the most planted variety in Mexico is Hass. The state of Michoacán is the main avocado producer with $80 \%$ out of the total, followed by other states such as Jalisco and state of Mexico. In Veracruz there is an area planted in the central part of the state that needs to be characterized. The objective of this study was to typify the avocado producing zone in the high mountain zone of Veracruz, Mexico. The avocado producing area in the high mountains region, Veracruz is made up of the municipalities of Alpatlahua, Calcahualco, Coscomatepex and Ixhuatlan del café. A survey was applied 
DOI: https://doi.org/10.5377/ribcc.v6i12.9941

Autor de correspondencia: juanmg_3@hotmail.com to producers using a semi-structured questionnaire with open and closed questions. The information analysis was carried out systematically with descriptive and multivariate analysis of components and correspondence, in addition to parametric and non-parametric analysis of the data for some variables. The results obtained showed groups of small producers, composed of $80 \%$ men. Most producers are literated, and they devoted themselves to activities other than avocado cropping. The avocado producer has a tacit knowledge of the crop management from the plantation stage to the tree production. Producers have experience in management of other crops because they have the purpose of obtaining additional income from other activities.

Keywords: Persea Americana, Fruit trees, Culture diagnosis.

\section{INTRODUCCIÓN}

El aguacate (Persea americana) es una fruta tropical con creciente consumo en el mundo (Gonzales, 2018), gracias a sus nutrientes y sus diferentes usos alimenticios. Este fruto se originó en las tierras Mesoamericanas, principalmente en la parte central de México, en la región norte de sudamérica y Guatemala, este se cultivaba antes de la llegada de los españoles. Su nombre "ahuacalt", proviene del nahualt, que significa "testículo de árbol” (Palacios, 2010;Saldívar, 2017). Su propagación puede ser por semilla o por injerto. Su fruto se conforma de tres capas, exocarpio (cascara), mesocarpio (pulpa) y endocarpio junto a la cubierta seminal (García et al., 1999). Este tiene un alto contenido de lípidos, ácidos grasos insaturados nutricionales como el oleico, palmítico, linoleico (Lu, 2009; López, 2012). Las variedades del aguacate que se distinguen son Hass, Fuerte, Bacon, Rinco, Zutano y Criollo selección Mexicana, estos materiales genéticos cultivados iniciaron la producción y comercialización en la década de los 50, 60 y 70 (Sánchez et al., 2013).

Los mercados europeos tienen el comercio mundial de aguacate, con un consumo del $60 \%$ por Francia, seguidos de los países como Alemania, Reino Unido e Italia. Sin embargo, México es uno de los países con mayor producción en los últimos 10 años con el 48.9\%, siendo Michoacán el primer productor con un $80 \%$, Jalisco con 6.6\%, el estado de México un 4.3\% y Morelos con 2.4\% (Escobedo, 2017; Rodríguez, 2017

El aguacate ha traído efectos positivos como incrementos de los ingresos en los mercados, el crecimiento de las empresas agroquímicas, asesorías y fuentes de empleos. México cuenta con una amplia diversidad de tipos de aguacate, al menos con 20 diferentes variedades, esto se debe a los diferentes factores ambientales y los cambios drásticos del clima que existentes en el territorio nacional, que pueden incidir en la etapa del desarrollo y la producción del cultivo de aguacate, como son la temperatura, viento y precipitación. Sin embargo, el aguacate se adapta en una amplia gama de suelos, desde los arenosos, hasta los arcillosos, siendo este último el más recomendado, con una disposición de profundidad de 0.8-1.0 m, con un pH aceptable de 5.5 hasta 6.5, para que el aguacate tenga una adecuada absorción de nutrimentos (Garrido, 2013; Dane, 2016). Existen tres variedades de aguacate la mexicana, antillana y guatemalteca, están difieren en su morfología, en la capacidad de adaptación edafoclimática. La variedad mexicana tiene condiciones tolerables al frio y es sensible a los suelos salinos, en comparación a los otros tipos de aguacates. Sus frutos son de menor tamaño, pero con un alto contenido de aceite (Campos et al., 2015).

El árbol de aguacate puede alcanzar $20 \mathrm{~m}$ de altura, su corteza es lisa y suave, presenta abundantes ramificaciones, que pueden ser susceptibles a quebraduras por el viento o quemaduras por sobreexposición

\section{NotAS DE AUTOR}


al sol. La primera cosecha de este cultivo ocurre a los 5 años y la cantidad de frutos producidos dependen de la variedad y el manejo que haya recibido la planta en su desarrollo (Campos et al., 2015). El tiempo de aprovechamiento de las especies forestales en las zonas aguacateras es de 40 a 80 años, dependiendo de las especies y de las condiciones agroecológicas. La producción del aguacate es a corto plazo, 2 a 3 años en arboles injertados y en zonas agroforestales en ciclos cortos hasta de 10 años, hasta largo plazo de 50 años. Sin embargo, para que las huertas duren la vida útil mencionada, se requiere tener un manejo eficiente a base de podas, análisis de suelo, control de insectos plagas, enfermedades, nutrición y riego (Bravo et al., 2009)

El aguacate en su cultivo presenta diferentes problemáticas como la presencia de plagas y enfermedades, y malezas, las cuales limitan la producción y con ello el comercio internacional, debido a los riesgos fitosanitarios y la exigencia de la calidad del producto, en los mercados (Hernández, 2013). El aguacate no alcanza su madurez de consumo en el árbol, debido a que este produce un inhibidor de la maduración que pasa al fruto por el pedúnculo. La determinación del momento de corte es un factor clave para garantizar que la fruta madure adecuadamente, optimizar la calidad de la fruta y minimizar las pérdidas de cosecha (Montero, 2006). Se estima que a nivel mundial existe una pérdida de postcosecha del 2-23\%, especialmente en la manipulación y el transporte de aguacate en países desarrollados y el $50 \%$ en países subdesarrollados. Por lo que es pertinente evaluar su comportamiento del fruto, sus características fisiológicas y fisicoquímicas durante la etapa de postcosecha (Márquez et al., 2014). Los huertos de aguacate requiere de ciertas atenciones en cuanto a su cultivo y cosecha, por lo que se necesita un manejo en las actividades de nutrición de suelos agrícolas, utilización apropiadamente del agua de riego y la aplicación del uso de plaguicidas y fertilizantes de origen sintético (Reyes et al., 2013). Por lo anterior el objetivo de esta investigación es tipificar la zona productora de aguacate en la zona de alta montaña de Veracruz, México.

\section{Materiales y MÉTODOS}

\section{Localización}

La zona productora de aguacate en la región de altas montañas, Veracruz, se caracteriza por estar integrado por los municipios de Alpatlahua, Calcahualco, Coscomatepex e Ixhuatlan del café (Figura 1). 


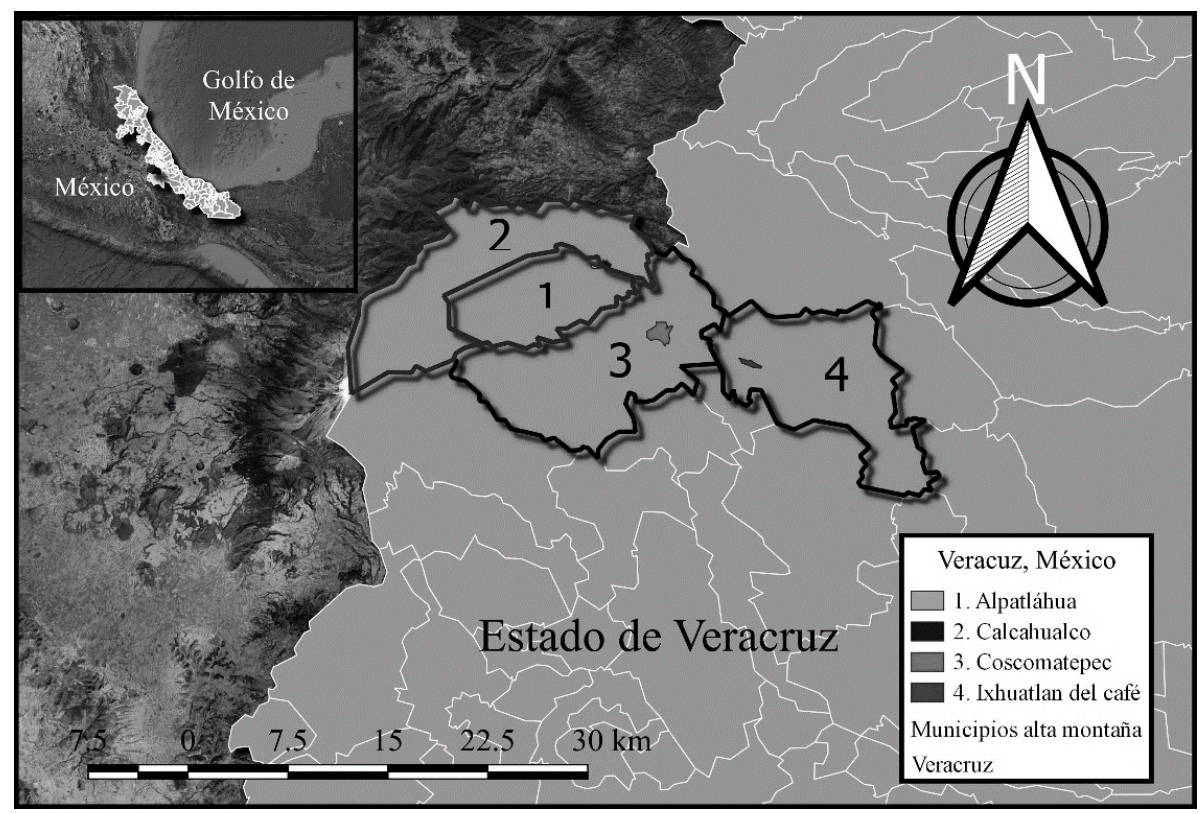

FIGURA 1.

Ubicación de los municipios productores de aguacate en la región de alta montañas de Veracruz (Elaborado por J. V. Megchún-García con información de INEGI, 2020)

\section{Método de diagnóstico}

Con base a la metodología de Frans (2002), el diagnostico consistió en una entrevista aplicada a una muestra del $40 \%$ de los productores de aguacate de manera individual y grupal; como parte del instrumento se utilizó un cuestionario con las siguientes características, los datos generales de la unidad de producción familiar, donde se describe las características de la familia y las condiciones económicas, además que se consideró la parte agroecológica y tecnológica en el proceso de producción del cultivo de aguacate. El cuestionario fue estructurado con preguntas abiertas y cerradas, para enriquecer de manera integral los datos socioeconómicos y técnicos de los informantes claves. El cuestionario fue integrado con 58 preguntas estructuradas en la siguiente forma, primero un apartado con los datos generales, unidad de producción, servicios de apoyo, información edafoclimática, datos del cultivo, tecnología de producción de aguacate, comercialización y la problemática. Para la aplicación de la encuesta se utilizaron técnicos agrícolas con experiencia en el sector agropecuario, el cual se les capacitó para validar el método de la encuesta semiestructurada; posteriormente, se procedió a definir a través de reuniones de grupos focales con los técnicos y productores, la estrategia para aplicar la encuesta en la zona de estudio de alta montaña del estado de Veracruz. Esta técnica consistió en que cada encuestador identificó al productor por localidad en cada municipio, y en los municipios donde la población de productores fue más grande se convocó a productores a través de grupos focales integrados $\geq 10$ individuos por grupo, esto permitió garantizar que la información recabada fuera específicamente a productores que se dedican al cultivo de aguacate, de acuerdo a estas características la encuesta 


\section{fue dirigida de tal manera que se cubriera la principal zona productora de aguacate por municipio, enmarcados como principales áreas de producción por la SEDARPA (Secretaria de Desarrollo Agropecuario, Rural y Pesca).}

\section{Análisis de la encuesta}

Para el análisis de la encuesta se agrupo la información en apartados, considerando los temas con mayor información relevante del cultivo de aguacate; los grupos fueron datos sociales, manejo del cultivo, características de los recursos naturales, tecnificación y se consideraron los datos más importantes en el manejo del cultivo. Para el análisis estadístico se realizó un análisis descriptivo de los datos el cual se consideró el análisis de Skewnes y Kurtosis y un análisis multivariado con dos factores y por correspondencia, y los datos cuantitativos fueron sometidos análisis de varianza y no paramétricos de Kruskal wallis, se utilizó el paquete estadístico Statistica versión 7.

\section{RESULTADOS Y DISCUSIÓN}

En los resultados obtenidos se puede observar que para la variable género la información recabada fue de tipo platicúrtica, respecto a las variables de escolaridad, localidad, municipio y las actividades que realizan diferentes al cultivo de aguacate, se observó una distribución de la información de tipo leptocúrtica (Cuadro 1). Para todas las variables se observó que en el coeficiente de asimetría se presentó una distribución extremadamente sesgada.

\section{CUADRO 1.}

Características de los productores de aguacate en la zona de altas montañas

\begin{tabular}{lllllll}
\hline \multicolumn{5}{c}{$\begin{array}{l}\text { Frecuencia Varianza } \\
\text { de la } \\
\text { moda }\end{array}$} & \multicolumn{3}{c}{$\begin{array}{l}\text { Desviación Error } \\
\text { estándar }\end{array}$} & $\begin{array}{l}\text { Skewness } \\
\text { Variable }\end{array}$ & & & & & \\
\hline Género & 28 & 0.16 & 0.40 & 0.06 & 1.57 & 0.48 \\
Escolaridad & 17 & 2.34 & 1.53 & 0.26 & 1.05 & -0.20 \\
Localidad & 8 & 28.37 & 5.33 & 0.90 & 0.52 & -1.16 \\
$\begin{array}{l}\text { Municipio } \\
\text { Realiza }\end{array}$ & 16 & 1.23 & 1.10 & 0.19 & 0.49 & -1.09 \\
$\begin{array}{l}\text { actividades } \\
\text { extras }\end{array}$ & 18 & 0.25 & 0.50 & 0.09 & 0.05 & -2.12 \\
\hline
\end{tabular}

Fuente: Elaboración propia

La edad de los productores de aguacate en la región de alta montañas es de 50 años (Cuadro 2) y tienen 20 años como productores dedicados al cultivo de aguacate, la unidad de producción familiar, está integrado por un grupo familiar por productor (Cuadro 2), esto podría estar correlacionada con la apropiación tecnológica, un caso especial en Puebla y Tlaxcala, registra que los productores más jóvenes tienen mayor innovación tecnológica, además de tener alto desempeño para otras actividades (Sangerman et al. 2014)

(Cuadro 3) 
CUADRO 2.

Descripción de los productores con respecto a su edad y años en el cultivo de aguacate

\begin{tabular}{|c|c|c|c|c|c|c|c|c|}
\hline Variables & Media & Mediana & $\begin{array}{l}\text { Frecuencia } \\
\text { de la } \\
\text { moda }\end{array}$ & Varianza & $\begin{array}{l}\text { Desviación } \\
\text { Estándar }\end{array}$ & $\begin{array}{l}\text { Error } \\
\text { Estándar }\end{array}$ & Skewness & Kurtosis \\
\hline $\begin{array}{l}\text { Edad del } \\
\text { productor } \\
\text { Años }\end{array}$ & 50.86 & 47.0 & 3 & 247.6 & 15.74 & 2.66 & 0.22 & -0.66 \\
\hline $\begin{array}{l}\text { como } \\
\text { productor } \\
\text { Unidad de }\end{array}$ & 20.63 & 12.0 & 5 & 407.4 & 20.18 & 3.41 & 0.70 & -0.60 \\
\hline $\begin{array}{l}\text { producción } \\
\text { familiar }\end{array}$ & 1.94 & 1.0 & 25 & 5.2 & 2.30 & 0.38 & 3.24 & 11.23 \\
\hline
\end{tabular}

Fuente: Elaboración propia

En la edad del productor de aguacate y los años dedicados al cultivo (Figura 2), se observó que existe una correlación entre productores de 40 años de edad, con una experiencia no mayor a 10 años en el cultivo (Figura 2). Por lo anterior los productores de aguacate son de una población caracterizados como ancianos con mayor experiencia y con conocimiento del manejo agronómico y la población de productores agrupados como jóvenes menores de 40 años es muy baja. Los productores suelen ser pequeños en superficie plantada, el rango es de 2 a 6 hectáreas plantadas en la zona de alta montaña de Veracruz, por lo que no existe diferencias significativas entre superficie plantada entre los municipios productores. Se tiene reporte de Michoacán, que los productores poseen un rango de 2.7 a 13.7 hectáreas, con rendimientos de 7.2 a 10.6 ton ha- 1 de aguacate, superiores a los productores de aguacate del estado de Veracruz (Montiel et al., 2008). En el estado de México, los productores de aguacates se catalogan como pequeños ya que tienen superficie inferior a 10 hectáreas, ante esta situación una forma de mejorar las condiciones de los

productores es trabajar a través de organizaciones, con el propósito de obtener apoyos de las diferentes estancias gubernamentales (Rubí et al., 2013).
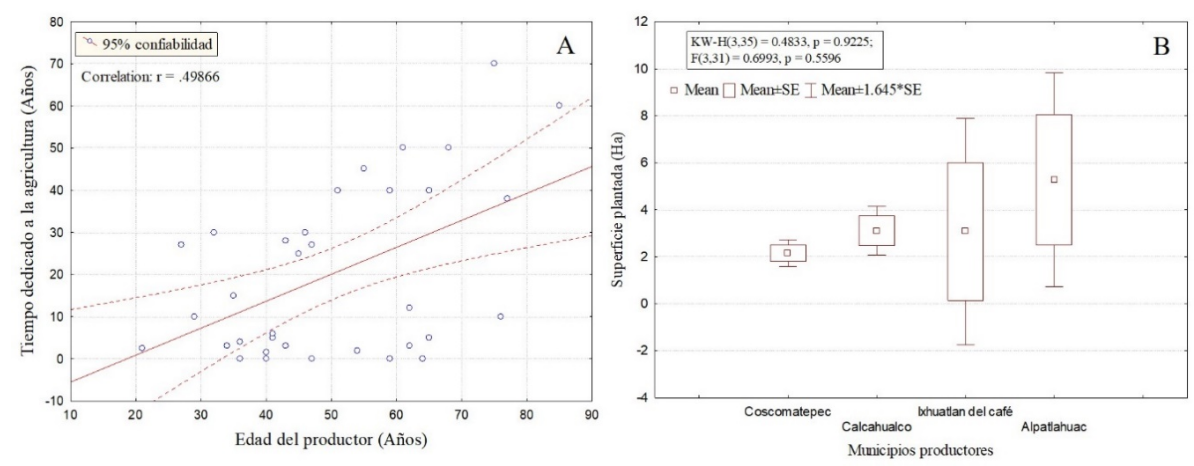

FIGURA 2.

Correlación entre edad del productor y tiempo dedicado al cultivo (A) y la superficie plantada de aguacate (B).

En la densidad de plantación del aguacate, los productores mencionaron que el municipio de Ixhuatlan del café no se reporta superficie plantada, en los municipios de Coscomatepec, Calcahualco y Alpatlahuac las densidades de plantación están entre rangos significativamente de 150 a 180 plantas/hectáreas (Figura 3). En el análisis entre componentes se observó que hay una relación entre las unidades de producción familiar con las actividades diferentes que realizan los productores del cultivo de aguacate; esto indica que los productores tienen otros ingresos que provienen de otros canales diferentes al cultivo de aguacate que les permite sostener a sus familias. 

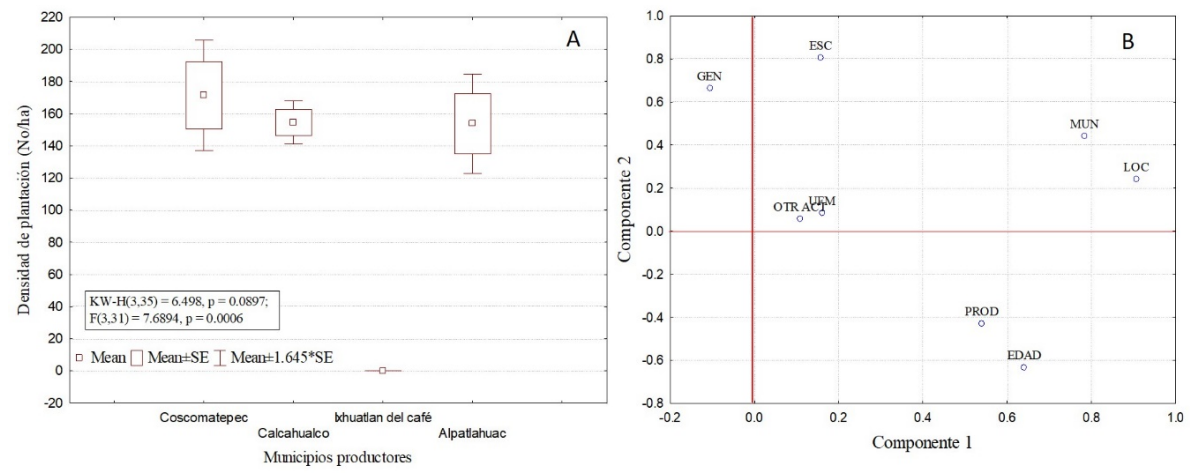

FIGURA 3.

Densidad de plantación (A) y características sociales de los productores de pitahaya (B)

En el manejo del cultivo de aguacate se consideró la densidad de plantación (DEN PL), el control de malezas (CON MAZ), los métodos de control de malezas (MET MAL), insectos plagas (PGL), dosis de los insecticidas (DOS INS) y el manejo de los insecticidas; como parte de la nutrición se consideró el análisis de suelo (AN SUL), la fertilización al establecimiento del cultivo (F EST), la fertilización química (F QUIM) y la fertilización orgánica (F ORG), el manejo de las dosis de fertilización química (DOS QUIM) y orgánica (DOS ORG), además del manejo de poda (POD), las variedades (VAR) y el rendimiento (REND) (Figura 4A). Se observó que el análisis del suelo tiene estrecha relación con la fertilización química en el cultivo y los métodos de control de malezas con las técnicas de podas manejadas en el aguacate y con el uso de las variedades al momento del establecimiento; además que la presencia de plagas está relacionado con la productividad del cultivo, esto quiere decir que el éxito del cultivo depende en gran parte del control fitosanitario durante los procesos de producción. En el caso de las características de la parcela, el uso de maquinaria está relacionado con el tipo de relieve de la zona productora, como la profundidad del suelo y la textura del suelo, debido a que estos factores suelen afectar el desarrollo del cultivo en sus distintas fases (Figura 4B). Se estima que el $80 \%$ de los suelos agrícolas en el mundo, son afectados con la erosión moderada o severa por el manejo de maquinaria pesada en condiciones de humedad alta, debido a que la maquinaria agrícola y los tractores ejercen una presión continua en el suelo (Gómez et al., 2018).
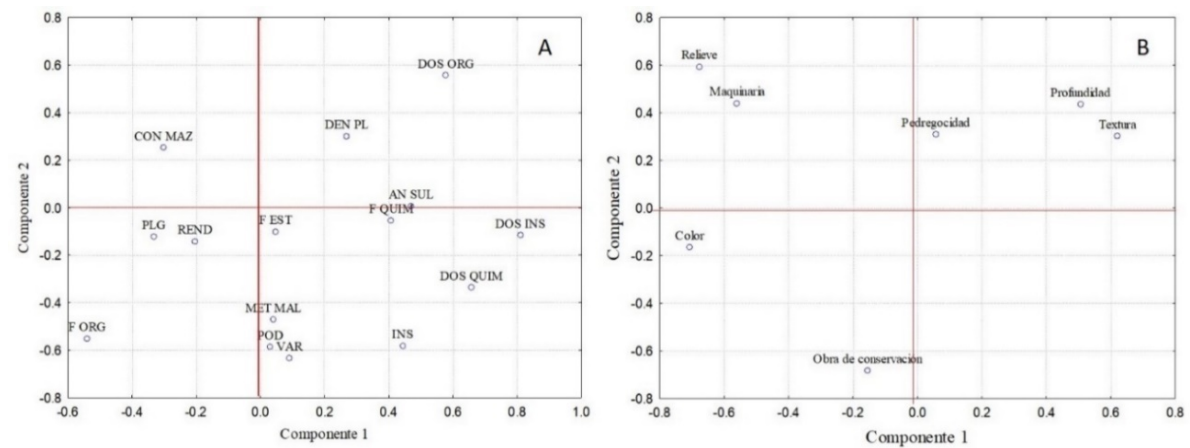

FIGURA 4.

Manejo del cultivo de aguacate (A) y características agroecológicas de las parcelas de los productores (B)

En el análisis de la fertilidad del suelo del cultivo de aguacate, (Figura 5) la baja fertilidad afecta principalmente el rendimiento y el desarrollo del cultivo, los productores mencionan que la presencia de ácaros en el cultivo, las poblaciones están relacionados entre sí, independientemente de la variedad de aguacate, caso similar con la presencia de hormigas y la plaga denominada barrenador del hueso, estas dos plagas podrían dar indicio, de que cuando se presenta uno de los insectos plaga es probable que exista presencia de los otros insectos plagas (Loya et al., 2003). En México, en el manejo fitosanitario se tiene la evidencia 
de la presencia de trips como una de las plagas que afecta la calidad y producción del fruto en la variedad de aguacate Hass, además de inhibir la fecundación de flores y provocar su caída; una de las recomendaciones más comunes adoptadas por los productores, sería el uso de plaguicidas ya que es común en el control de trips (Thrips tabaci Lindeman; Thysanoptera: Thripidae), pero con esta actividad se incurre a romper el equilibrio de las cadenas tróficas, esto provocaría la presencia de 40 especies de trips en el cultivo de aguacate (Cambero et al., 2009).
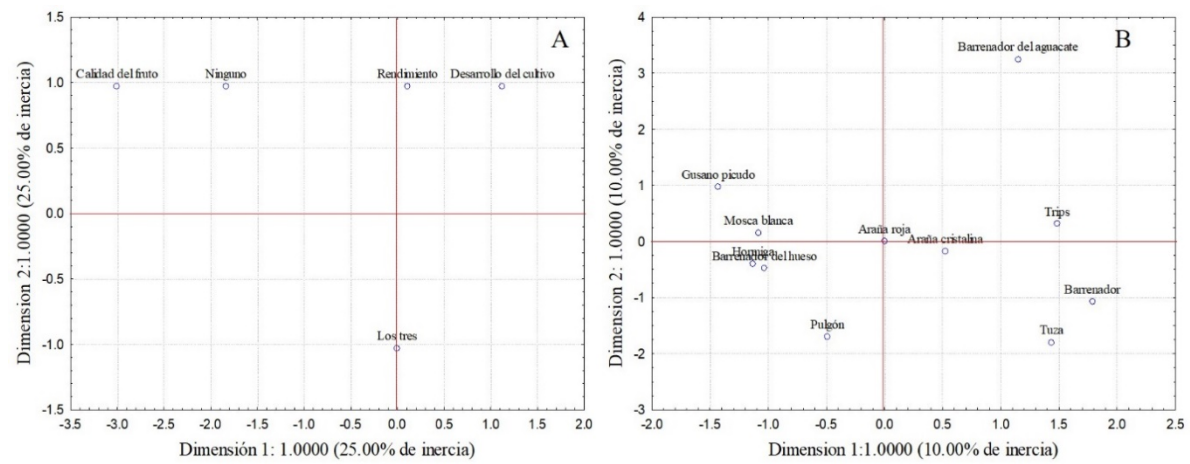

FIGURA 5.

Efectos de la baja fertilidad en el cultivo (A) y presencia de plagas en cultivo de aguacate B)

Para la nutrición del cultivo de aguacate, los productores utilizan 16 fuentes de fertilizantes con diferentes concentraciones, los fertilizantes con el nombre comercial Yara Mila Complex y Yara Hydran (Durlak et al., 2019), son manejados en dosis altas de fertilizantes (sintéticos y orgánicos) y el aporte de

nutrimentos al año. Necesario para caracterizar los rendimientos con los siguientes elementos de S-MgO$\mathrm{B}-\mathrm{Fe}-\mathrm{Mn}$ y Zn. En el manejo de las diferentes fuentes de fertilizante orgánico; las fuentes de estiércoles y

hojarasca compostada los productores utilizan la dosis de $10 \mathrm{~kg}$ por árbol (Figura 6A). En la fertilización al momento de la plantación, esta se realiza de manera manual y se utilizan seis fuentes de origen animal, además, se observó diferencias significativas al analizar las dosis de los fertilizantes orgánicos; por ejemplo, el fertilizante estiércol de vacuno es diferente en su composición con la dosis de fertilización de abono de borrego, abono de caballo y el uso de hojarasca, la dosis más altas de abono orgánico que manejan los productores de acuerdo a sus experiencias en sus cultivos es mayor a $25 \mathrm{~kg}$ por árbol/año (Figura 4b). En Michoacán utilizan para la nutrición fuentes a base de lombricomposta, composta orgánica y algunos derivados del pescado, estos productos tienen mayor efecto en el cuajado del fruto de aguacate (formación de frutos), mientras que el uso de un fermentado orgánico produce mayor amarré de fruto y el rendimiento del árbol es de $160 \mathrm{~kg}$ por árbol, pero el uso de lombricomposta genera un rendimiento de $104 \mathrm{~kg}$ por árbol (Tapia et al., 2014). De acuerdo a la FAO para el cultivo de aguacate no se especifica el tipo de suelo, solo se considera la condición física, relacionada directamente con el drenaje y la aireación del suelo (Rodríguez, 1980). Pero en el estado de Michoacán, México el tipo de suelo donde prospera mejor el aguacate es el andosol, considerado como suelo con mejores condiciones adafloclimáticas y con una eficiente permeabilidad, regularmente existen 10 tipos de suelos donde prospera mejor el aguacate entre ellos los semicálidos, cálidos y templados (Gutiérrez et al., 2010). 

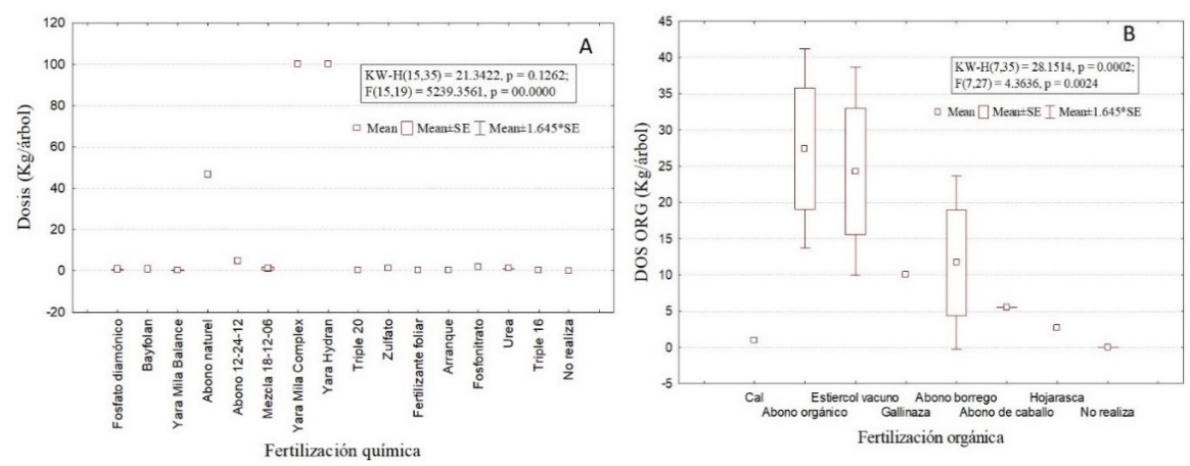

FIGURA 6.

Manejo de fertilización química (A) y orgánica en el cultivo de aguacate (B)

En el manejo de los insecticidas en el cultivo de aguacate existen 18 productos químicos para el control de plagas, entre los que destacan los insecticidas monocrotofos y azufre elemental, usados en mayores concentraciones. Existen algunos productores que han manejado en su cultivo el uso de cal en dosis de $50 \mathrm{~kg}$ por árbol, pero se reportan para Colombia en aguacate la dosis de 1 a $10 \mathrm{~kg}$ de cal por árbol en pH de 5.5 a 6.5 (Serna et al., 2012); también, utilizan la abamectina para el control de ácaros (Opiliocarida, Mesostigmata, Ixodida, Trombidiformes y Sarcoptiformes). Se observó diferencias significativas entre dosis de insecticidas por árbol, entre ellos destacan el insecticida monocrotofos, azufre elemental y cal en comparación con los demás ingredientes activos (Figura 7). Esta actividad en el cultivo de aguacate es importante debido a que marca la pauta para ingresar al mercado internacional, tal como es el caso en un estudio de redes con productores del estado de Michoacán donde ellos realizan de 3 a 15 innovaciones (Guerra et al., 2012).

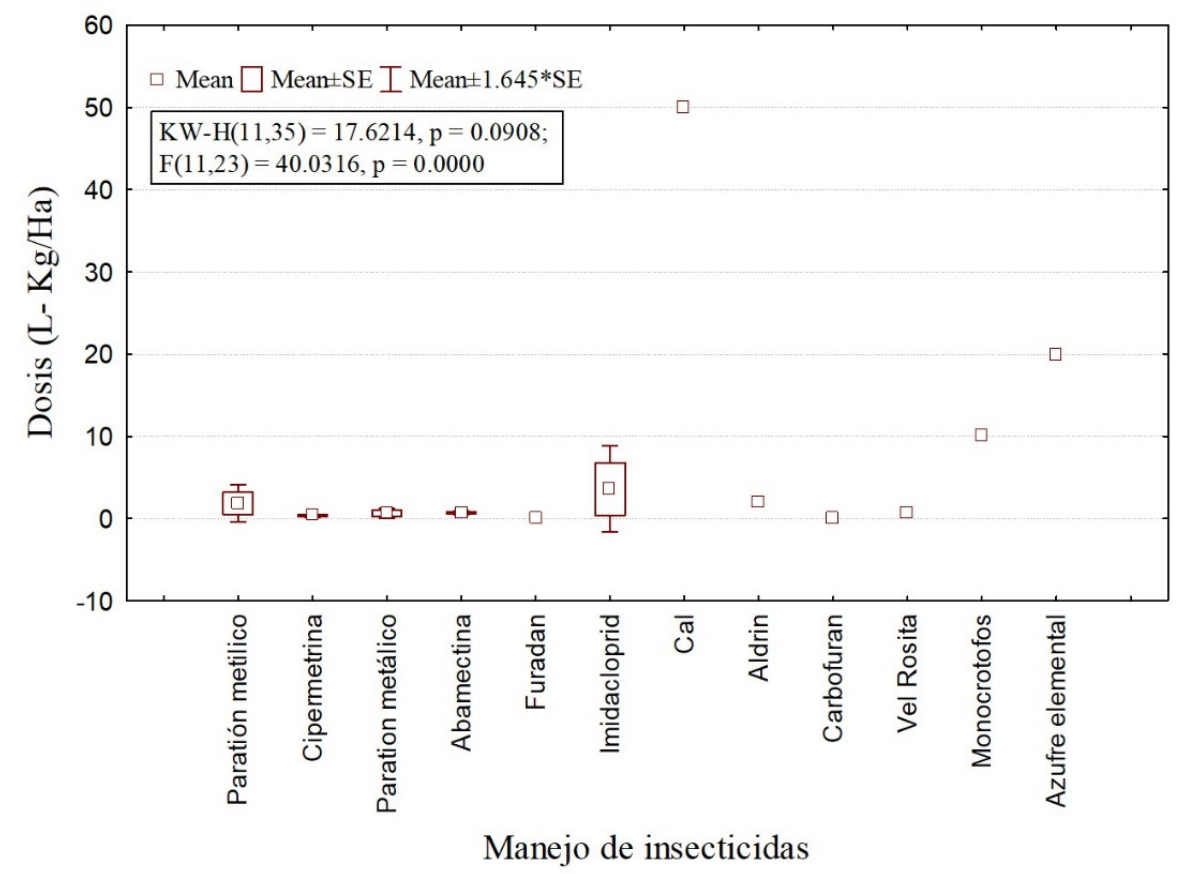

FIGURA 7.

Manejo de los insecticidas y sus dosis en el cultivo aguacate

La asociación del aguacate con otros cultivos es importante para la sostenibilidad agrícola del productor o agricultor. El manejo del cultivo del aguacate está íntimamente relacionado con las actividades diferentes al cultivo de aguacate, ya que el ingreso económico por otras labores permite sustentar las familias de los 
campesinos. El $22.8 \%$ de los productores asocia el cultivo de aguacate con maíz, mientras el $37.1 \%$ de los productores menciona que no asocia el cultivo de aguacate; pero el $20 \%$ de los productores mencionó que se dedica al cultivo de maíz y frijol, situación que amerita que los productores establecen de manera intensiva o en el esquema de monocultivo y el $14.3 \%$ de los productores se dedica al monocultivo de maíz, seguido del $11.4 \%$ de los productores que realizan la actividad de la albañilería (Cuadro 4). Las prácticas agroforestales en el cultivo de aguacate son más productivas y económicamente más rentable que los sistemas de monocultivo, además que los sistemas de asociación de aguacate pueden incrementar la productividad y la rentabilidad del cultivo de aguacate y contribuir al tema ambiental como económico; por lo que sería importante focalizar esfuerzo en este tema para impulsar la bioeconomía en el cultivo de aguacate a través de los procesos de asociación (Montiel et al., 2008). En Montes María, Colombia, los productores de aguacate se ven afectados en la comercialización al no tener grandes beneficios o ganancias, quedándose la mayor ganancia con los intermediarios (Méndez et al., 2015). Unos de los beneficios de la asociación de los cultivos es la biodiversidad de especies, además que los enemigos naturales son más abundantes en los policultivos, por lo que se promueve un control de plagas de manera natural (Loya et al., 2003). Con la asociación de los cultivos se mejora la fertilidad del suelo, permite proteger el suelo de la erosión, no se depende del monocultivo y se mejora la bioeconomía de los cultivos (Rebolledo et al., 2019). 
CUADRO 4.

Características de los productores de aguacate

\begin{tabular}{|c|c|c|c|}
\hline $\begin{array}{l}\text { Asociación del } \\
\text { aguacate }\end{array}$ & $\begin{array}{l}\text { Productores } \\
\text { (\%) }\end{array}$ & $\begin{array}{l}\text { Actividades } \\
\text { extras }\end{array}$ & $\begin{array}{l}\text { Productores } \\
(\%)\end{array}$ \\
\hline $\begin{array}{l}\text { Chile serrano } \\
\text { (Capsicum annuum } \\
\text { var. Acuminatum) }\end{array}$ & 5.7 & Maíz (Zea mays) & 14.3 \\
\hline Maíz (Zea mays) & 28.2 & $\begin{array}{l}\text { Café (Coffea sp.), } \\
\text { frijol }\end{array}$ & 5.7 \\
\hline Café (Coffea sp.) & 5.7 & Ama de casa & 5.7 \\
\hline $\begin{array}{l}\text { Maíz (Zea mays), } \\
\text { rábano (Raphanus } \\
\text { sativus) }\end{array}$ & 2.8 & $\begin{array}{l}\text { Maí (Zea mays) y } \\
\text { frijol (Phaseolus } \\
\text { vulgaris) }\end{array}$ & 20.0 \\
\hline No asocia & 37.1 & Aguacate & 2.8 \\
\hline $\begin{array}{l}\text { Papa (Solanum } \\
\text { tuberosum) } \\
\text { Durazno (Prunus } \\
\text { pérsica), frijol }\end{array}$ & 2.8 & Café (Coffea sp.) & 8.6 \\
\hline $\begin{array}{l}\text { gordo (Phaseolus } \\
\text { dumosus) y chile } \\
\text { cera (Capsicum } \\
\text { pubescens) }\end{array}$ & 2.8 & Zapateria & 2.8 \\
\hline $\begin{array}{l}\text { Durazno (Prunus } \\
\text { pérsica) }\end{array}$ & 2.8 & $\begin{array}{l}\text { Maiz (Zea mays) y } \\
\text { chayote (Sechium } \\
\text { edule Jacq) }\end{array}$ & 2.8 \\
\hline $\begin{array}{l}\text { Chile cera } \\
\text { (Capsicum } \\
\text { pubescens) }\end{array}$ & 2.8 & Agropecuarios & 5.7 \\
\hline $\begin{array}{l}\text { Maíz (Zea mays), } \\
\text { frijol (Phaseolus } \\
\text { vulgaris) }\end{array}$ & 2.8 & Albañilería & 11.4 \\
\hline $\begin{array}{l}\text { Café (Coffea sp.) } \\
\text { frijol (Phaseolus } \\
\text { vulgaris) }\end{array}$ & 2.8 & $\begin{array}{l}\text { Chayote (Sechium } \\
\text { edule Jacq) }\end{array}$ & 2.8 \\
\hline \multirow[t]{4}{*}{$\begin{array}{l}\text { Chayote (Sechium } \\
\text { edule Jacq) }\end{array}$} & \multirow[t]{4}{*}{2.8} & $\begin{array}{l}\text { Papa (Solanum } \\
\text { tuberosum) }\end{array}$ & 5.7 \\
\hline & & $\begin{array}{l}\text { Chile (Capsicum } \\
\text { sp.) }\end{array}$ & 5.7 \\
\hline & & Maíz (Zea mays), & 2.8 \\
\hline & & $\begin{array}{l}\text { Maí (Zea mays) y } \\
\text { tomate (Solanum } \\
\text { lycopersicum) }\end{array}$ & 2.8 \\
\hline
\end{tabular}

Fuente: Elaboración propia

Fuente: Elaboración propia

\section{CONCLUSIONES}

La población de productores de aguacate en la zona de alta montaña está integrada por los municipios de Alpatlahua, Calcahualco, Coscomatepex e Ixhuatlan del café. Los productores tienen edad promedio de 50 años y poseen 20 años de experiencia en el cultivo de aguacate, el 70\% de los productores establece la variedad Hass, en densidades de 125 plantas ha-1. El productor aguacatero posee conocimiento tácito en el manejo del cultivo, desde la etapa plantación hasta la producción del cultivo; además que no se dedica únicamente al cultivo de aguacate, ya que posee experiencia en el manejo agronómico de otros cultivos, esta actividad se realiza con la finalidad de obtener otros ingresos adicionales. Otras de las características de los productores aguacateros, es que no está muy tecnificado con respecto al manejo de equipos; también conoce e identifica muy bien los problemas productivos y su relación como la baja fertilidad de los suelos. Es 
importante mencionar que se consideran a este grupo de productores como pequeños, ya que la superficie plantada por productor no supera las 10 hectáreas.

\section{LiTERATURA CITADA}

Bravo E. M., Sánchez P. J. L., Vidales F. J. A., Saenz R. J. T., Chávez L. G. J., Madrigal H. S., Muños F. J. H., Tapia V. L. M., Orozco G. G., Alcantar R. J. J., Vidales F. I., Venegas G. E., 2009. Impactos ambientales y socioeconómico del cambio de uso del suelo forestal a huertos de aguacate en Michoacán. (INIFAP) Instituto Nacional de Investigación Forestales, Agrícola y Pecuaria. Centros de investigación regional pacifico del centro. http://www.inifapcirne.gob.mx/Revistas/Archivos/libro_aguacate.pdf

Cambero C. O. J., Johansen N. M., García M. O., Carvajal C. C. R., Isiordia A. N., Cantu S. M., 2009. Thrips fitófagos en huertas de aguacate cv. Hass en Nayarit, México. Brenesia, 71(72): 61-64.

Campos B., Calderón E., Gonzales J., Barcelo A., Sarmiento D., Gonzales M. J., Medina D., Barea A., Guirando E., Ramón B. J., 2015. El cultivo del aguacate. Instituto de investigación y formación agraria y pesquera. Consejería de agricultura, pesca y desarrollo rural. file:///C:/Users/roosy/Downloads/Curso\%20el\%20cultivo \%20del\%20aguacate_Resumenes\%20ponencias\%20(1).pdf.

Dane, 2016. Cultivo de aguacate Hass (Persea americana Mill; Persea nubigena var. Guatemalensis x Persea americana var. drymifolia), plagas y enfermedades durante la temporada de lluvias. Insusmos y factores asociados a la producción agropecuaria. 50. 1-102. https://www.dane.gov.co/files/investigaciones/agropecuario/sipsa/ Bol_Insumos_ago_2016.pdf

Durlak W., Marcinek B., Szmagara M., Dudkiewicz M., Konopinska M. A., 2019. Effect of selected preparations of tardiva panicled hydrangea (Hydrangea paniculata Siebold) depending on the irrigation frequency. Acta Sci. Pol. Hortorum, 18(2): 39-51. https://doi.org/10.24326/asphc.2019.2.5

Frans, G. 2002. 80 herramientas para el desarrollo participativo: diagnóstico, planificación monitoreo, evaluación/ Frans Geifus-San Jóse, C.R.: IICA. 217 P.

García F. J. A., Ramos G. M. del R., Mora G. J., 1999. Estructura de la semilla de aguacate y cuantificación de la grasa extraída por diferentes técnicas. Centro de investigación y asistencia en tecnología y diseño del estado de Jalisco. 124-128. https://www.chapingo.mx/revistas/revistas/articulos/doc/rchshV1104.pdf.

Garrido R. E., Noriega D., Gonzales M. R., 2013. Áreas potenciales para el cultivo del aguacate (Persea americana L.) cultivar Hass en el estado de Guerrero, México. INIFAP. Campo experimental de investigación forestal acuícola y pecuario. Reseachgate. file://C:/Users/roosy/Downloads/Aguacatecont7.pdf

Gómez C. N., Villagra M. K., Solorzano Q. M., 2018. La labranza mecanizada y su impacto en la conservación del suelo (revisión de literatura). Tecnología en marcha. 31(1):170-180. https://doi.org/10.18845/tm.v31i1.3506

Gonzales S. C. E., 2018. Cultivo del aguacate (Persea americana Miller). Centro nacional de tecnología agropecuaria y forestal. Ministerio de agricultura y ganadería. 7-24. http://centa.gob.sv/docs/guias/frutales/ Guia\%20Centa_Aguacate\%202019.

Guerra P. J., Ávila M. L. A., Ponce M. F., 2012. Caracterización del sistema producto aguacate en los reyes, Michoacán, $2^{\circ}$. Congreso internacional de desarrollo comunitario At. Pátzcuaro.

Gutiérrez C. M., Lara C. Ma. B. N., Guillén A. H., Chávez B. A. T., 2010. Agroecología de la franja aguacatera en Michoacán, México. Interciencia. 35(9): 647-653.

Hernández G. H., Ramírez G. M. E., Arévalo G. L., 2013. Histología del daño en fruto y ramas de aguacate "Hass" por escamas aramadas (Hemiptera: Diaspidiae). Revista Mexicana de ciencias agrícolas. 5 (4):739-751. file:/// C:/Users/roosy/Downloads/ArticuloRuben.pdf. https://doi.org/10.29312/remexca.v4i5.1172

INEGI. 2020. https://www.inegi.org.mx/mapasitio/.

López G. G., Medina T. R., Guillem A. H., Ramírez G. L., Aguilar C. J., Valdivia R. G. M., 2012. Características fenotípicas de hojas y fruto en selecciones de aguacate criollo de clima subtropical en el estado de Nayarit. Unidad académica de agricultura, universidad autónoma de Nayarit, México. http://fuente.uan.edu.mx/ publicaciones/04-10/6.pdf. 
Loya R. J. G., García-H. J. L., Ellington J. J., y Thompson, D. V. 2003. Impacto de la asociación de cultivos en la densidad de insectos hemípteros entomófagos. Interciencia, 28(7), 415-420.http://ve.scielo.org/scielo.php? script=sci_arttext\&pid=S0378-18442003000700010\&lng=es\&tlng=.

Lu Q., Zhang Y., Wang Y., Lee R., 2009. Identificación y caracterización de frutos de selecciones de aguacate criollo en el clima cálido de Nayarit, México. Journal of agriculturall and food chemistry. 57 (104):08-13

Márquez C. J., Yepes P. D., Sánchez L., Osorio J. A., 2014. Cambios fisicoquímicos del aguacate (Persea americana Mill. cv. "Hass") en poscosecha para dos municipios de Antioquia. (19):1. 32-37. file://C:/Users/roosy/ Downloads/DialnetCambiosFisicoquimicosDelAguacatePerseaAmericanaMil-4994548\%20(3).pdf https:// doi.org/10.21897/rta.v19i1.723

Méndez P. M. C., Humanez M. U. N., Pérez R. J. A., Bertel O. C. A., 2015. Estrategia de distribución de la cadena productiva del aguacate en los montes de maría. Perspectiva, socieconómica, 2. 105-118. https:// doi.org/10.21892/24627593.228

Montero C. M., Cerdas A. M. M, Diaz C. E., 2006. Manual de manejo pre y poscosecha de aguacate. Ministerio de agricultura y ganadería centro de investigación agronómica. Universidad de costa rica. Consejo nacional de producción. http://www.mag.go.cr/bibioteca_virtual_ciencia/aguacate-2006.pdf.

Montiel A. G., Krishnamurthy L., Vázquez A. A. y Uribe G. M., 2008. Opciones agroforestales para productores de aguacate. Terra Latinoamericana. 26 (1): 85-90.

Palacios S. M. B., 2010. El aguacate y sus diferentes aplicaciones en 25 recetas. Universidad de cuenca facultad de ciencias de la hospitalidad carrera de gastronomía. 4-181. https://dspace.ucuenca.edu.ec/ bitstream/123456789/1626/1/tgas14.pdf.

Rebolledo M. L., Megchún G. J. V., Rebolledo M. A., Orozco C. D. M., 2019. Asociación de frutales de limón persa (Citrus latifolia) y palma de coco (Cocos nucifera L.) con el aporte de materia seca por cultivos anuales. Revista Iberoamericana de Bioeconomia y Cambio Climático. 5 (10): 2410-7980. https://doi.org/10.5377/ ribcc.v5i10.8968

Reyes J. C., Rubi A. M., Aguilar M. J. J., 2013. Manejo orgánico en el cultivo de aguacate. Fundación Salvador Sánchez Colin. http://www.avocadosource.com/Journals/CICTAMEX/CICTAMEX_1995/Ecol_1_95.pdf.

Rodríguez C. J., Escobedo R. A., Lugo M. O. Y., 2017. Inocuidad del aguacate. Unidad de servicios analíticos y meteorológicos. Centro de investigación y asistencia en tecnología y diseños del estado de Jalisco A. C. 165-186. https://ciatej.repositorioinstitucional.mx/jspui/bitstream/1023/634/1/cap\%20IInocuidad\%20Aguacate.pdf.

Rodríguez Z. E., 1980. El cultivo de frutales en obras de conservación de suelos. Ordenación integrada de cuencas hidrográficas. FAO. Tegucigalpan, Honduras. 61 p.

Rubí A. M., Franco M. A. L., Rebollar R. S., Bobadilla S. E. E., Martínez de la C. I., Siles H. Y., 2013. Situación actual del cultivo de aguacate (Persea americana Mill) en el estado de México, México. Tropical and subtropical agroecosystems. 16:93-101.

Saldívar I. P., 2017. Producción de cultivos Perennifolios, cultivo aguacate (Persea americana Miller). Campus Universitario El Cerrillo. Universidad Autónoma del Estado de México, Facultad de Ciencias Agrícolas. http://ri.uaemex.mx/bitstream/handle/20.500.11799/66699/Cultivo_aguacate_Perennifolios.pdf? sequence $=3 \&$ is Allowed $=y$

Sánchez S. C., Mijares O. P., López L. L., Barrientos P. A. F. 2013. Historia del aguacate en México. Departamento de fitotecnia, universidad autónoma de Chapingo. 171-187. http://www.avocadosource.com/journals/cictamex/ cictamex_1998-2001/CICTAMEX_1998-2001_PG_171-187.pdf.

Sangerman J. D. Ma., Larqué S. B. S., Omaña S. J. M., Shwenstesius de R. R., Navarro B. A. 2014.Tipología del productor de aguacate en el estado de México. Revista Mexicana Agrícolas. 5(6):1081-1095. https:// doi.org/10.29312/remexca.v5i6.892

Serna S., Montoya B., Osorio W., 2012.Monitoreo del pH y fósforo soluble en la rizosfera de árboles de aguacate en el norte y oriente Antioqueño. Suelos Ecuatorianos. 42 (2): 186-190. 
Jeremías Nataren-Velazouez, et al. Caracterización productiva del aguacate (Persea americana) en l...

Tapia V. L. M., Larios G. A., Hernández P. A., Guillén A. H., 2014. Nutrición orgánica del aguacate cv. Hass y efecto nutrimental y agronómico. Revista Mexicana de Ciencias Agrícolas. 5(3): 463-472. https://doi.org/10.29312/ remexca.v5i3.950

Escobedo, N., \& Oliver, G. (2017). The lymphatic vasculature: its role in adipose metabolism and obesity. Cell metabolism, 26(4), 598-609. 